Article

\title{
Consistency of Control Performance in 3D Overhead Cranes under Payload Mass Uncertainty
}

\author{
Uyen Tu Thi Hoang ${ }^{1}$, Hai Xuan Le ${ }^{2}$, Nguyen Huu Thai ${ }^{3}$, Hung Van Pham ${ }^{2}$ and Linh Nguyen ${ }^{4, *}$ \\ Department of Automation, Industrial University of Ho Chi Minh City, Ho Chi Minh City 70000, Vietnam \\ Department of Instrumentation and Control Engineering, Hanoi University of Industry, Hanoi 1000, Vietnam \\ Faculty of Electrical Engineering, Vinh University of Technology Education, Nghe An 43000, Vietnam \\ 4 School of Science, Engineering and IT, Federation University Australia, Churchill 3842, VIC, Australia \\ * Correspondence: 1.nguyen@federation.edu.au; Tel.: +61-3-5122-6129
}

Received: 26 March 2020; Accepted: 15 April 2020; Published: 16 April 2020

check for updates

\begin{abstract}
The paper addresses the problem of effectively and robustly controlling a 3D overhead crane under the payload mass uncertainty, where the control performance is shown to be consistent. It is proposed to employ the sliding mode control technique to design the closed-loop controller due to its robustness, regardless of the uncertainties and nonlinearities of the under-actuated crane system. The radial basis function neural network has been exploited to construct an adaptive mechanism for estimating the unknown dynamics. More importantly, the adaptation methods have been derived from the Lyapunov theory to not only guarantee stability of the closed-loop control system, but also approximate the unknown and uncertain payload mass and weight matrix, which maintains the consistency of the control performance, although the cargo mass can be varied. Furthermore, the results obtained by implementing the proposed algorithm in the simulations show the effectiveness of the proposed approach and the consistency of the control performance, although the payload mass is uncertain.
\end{abstract}

Keywords: 3D overhead crane; adaptive control; neural network; load uncertainty; sliding mode control; Lyapunov theory

\section{Introduction}

A 3-dimensional (3D) overhead crane, also called a gantry crane, increasingly plays a crucial role in lifting and transporting goods and containers at seaports or manufactories, where its operations are required to be extremely precise and safe [1]. Nonetheless, an overhead crane is modeled as an under-actuated mechanical system where the number of control signals it has is less than the number of its degrees of freedom (DoF). The under-actuation can result in unexpected swings of the payload. More importantly, given the uncertainty of the payload mass, the unexpected swings can endanger the lives of people and demolish the surrounding machineries and goods [2]. In order to guarantee safety and precision in operating a gantry crane, the reliability and stability of the control law for the crane are indispensable indicators in designing a controller.

Many control strategies for a crane have been proposed in the past decades. A fairly simple controller can be presented by the open-loop control law, where implementing it on a crane is straightforward, since no sensor is required [3-6]. Nevertheless, the overhead crane relying on the open-loop controller can be quite sensitive to external disturbances such as rain and wind when it is operating in outdoor environments [7]. On the contrary, a closed-loop controller can use readings from the integrated sensors to adjust the actuators of the crane so that its operations are stabilized, even under external noises or system uncertainties [8]. The first example of a closed-loop controller is the proportional-derivative control law based on LaSalle's invariance set theorem, which was proposed for 
a two DoF crane [9]. Similarly, a nonlinear two feedback control scheme was proposed [10] to reduce a crane's swing angles when an object is transported to a new place. The authors of the work [11] linearized the partial feedback of the nonlinear dynamics on a 2-dimensional (2D) crane, which allowed them to design a controller to instantly control both the trolley position and sway angles. By extending the 2D nonlinear partial feedback control approach from [11], Le et al. [12,13] designed asymptotically stabilized control strategies for a 3D gantry crane.

In fact, it is highly-expected that a crane can be controlled robustly due to its nonlinearities and uncertainties, including its under-actuated mechanism, its uncertain system parameters, and the external disturbances from the working environment. To this end, some other works have proposed to employ the sliding mode control (SMC) technique [14-22] in designing a robust controller for a gantry crane. For instance, the authors in $[17,19]$ proposed a control law with a sliding surface linearly-constituted from the actuated state sliding subsurface and the under-actuated state errors. Likewise, Qian et al. $[18,23]$ utilized the state errors as an intermediate variable to develop the second-order sliding surface. However, the hierarchical SMC (HSMC) technique has attracted more attention from researchers, practitioners and engineers who have designed a robust controller for an under-actuated system. Fundamentally, the HSMC method is formed by multiple layers of the sliding surfaces. In [24], the authors proposed that the first-level sliding surface is constructed by the state variable and their weights while the second-level one is simply linearly combined by multiple first-order layers [24]. Some other researchers designed the HSMC scheme in a different manner. For instance, Wang et al. [25] employed the sliding surfaces of the first and second subsystems to build the second-order sliding surface; then, they utilized the formed second-order sliding surface and the sliding surface of the third subsystem to build the third-order sliding surface. The construction continued until the last subsystem, where they proved that the hierarchical sliding surfaces were stable.

The main drawback when employing the SMC method to design a controller, especially for an overhead crane, is that all the model parameters are required to be deterministic. However, it is transparent that the parameters on a crane system are highly uncertain and nonlinear, particularly under the external disturbances. To address this impracticality of using the SMC control approach, some adaptive strategies have been proposed to estimate the parameters of a crane online. For instance, authors have [26] employed the Lyapunov theory to derive an adaptive technique to estimate the unknown parameters of an overhead crane. Park et al. [27], proposed an observer-based fuzzy logic to present the uncertainties and nonlinearities of the crane actuators. In addition, an adaptive SMC law based on the fuzzy wavelet neural networks was proposed for a 3D gantry crane as discussed by other authors [28]. In our previous works [29,30], we introduced the adaptive HSMC schemes for the 2D and 3D overhead cranes, respectively, where we employed the fuzzy observer and radial basis function network to adaptively estimate the unknown and uncertain crane parameters. Nevertheless, to the best of our knowledge, none of the previous adaptive control schemes for the 3D overhead crane considered the consistency of the control performance of the closed-loop control system when the payload mass is uncertain.

As a result, in this work, in order to effectively and robustly control a 3D overhead crane even under uncertainty of the payload mass, we first propose to employ the radial basis function neural network to adaptively estimate the unknown and uncertain system kinetic parameters of the controller, which is designed based on the SMC technique. More importantly, we then propose to incorporate the adaptation mechanisms [31] into the control law that allows the controller to adaptively estimate and update the payload mass online in order to compute its other unknown parameters. The proposed closed-loop control system is then mathematically proven to be stable by the using the Lyapunov theory, which consolidates that the 3D gantry crane can robustly operate under uncertain payload mass. The proposed algorithm was evaluated in the synthetic simulation experiments, where the obtained results show that the control performance was consistent, although the payload mass varied.

The remainder of this paper is arranged as follows: Section 2 presents how to design a SMC controller for a 3D overhead crane. Section 3 presents the proposed adaptive methods to estimate 
the unknown and uncertain system parameters, where the payload mass is updated online, and also discusses the stability of the closed-loop control system for a 3D overhead crane. Section 4 provides discussions of the validated results obtained by the proposed approach before conclusions are drawn in Section 5.

\section{A Sliding Mode Controller for Uncertain Payload-Mass 3D Overhead Cranes}

\subsection{D Overhead Crane Dynamic Model}

Practically, a 3D gantry crane comprises of three main components as presented in Figure 1: a bridge, which can move to the left or right; a trolley sliding forward or backward on the bridge; and a hoist to carry objects or cargo. Let us denote the trolley mass as $m_{c}$, the bridge lumped mass as $m_{r}$, the cargo mass as $m$ and the length of the hoist cable is $l$. For the purposes of simplicity, we assume that the hoist cable length is fixed. The forces exerted on the trolley in the $x$-direction and $y$-direction are defined as $u_{x}$ and $u_{y}$, respectively. We also denote $\theta_{x}$ as the sway angle of the payload and $\theta_{y}$ as the indirect dependent parameter of the oscillation component of the crane. Without a loss of generality, it is assumed that the static friction in the gantry crane is defined, and the mass of the hoist cable is trivial and ignored. Both the trolley and cargo are considered as the material particles.

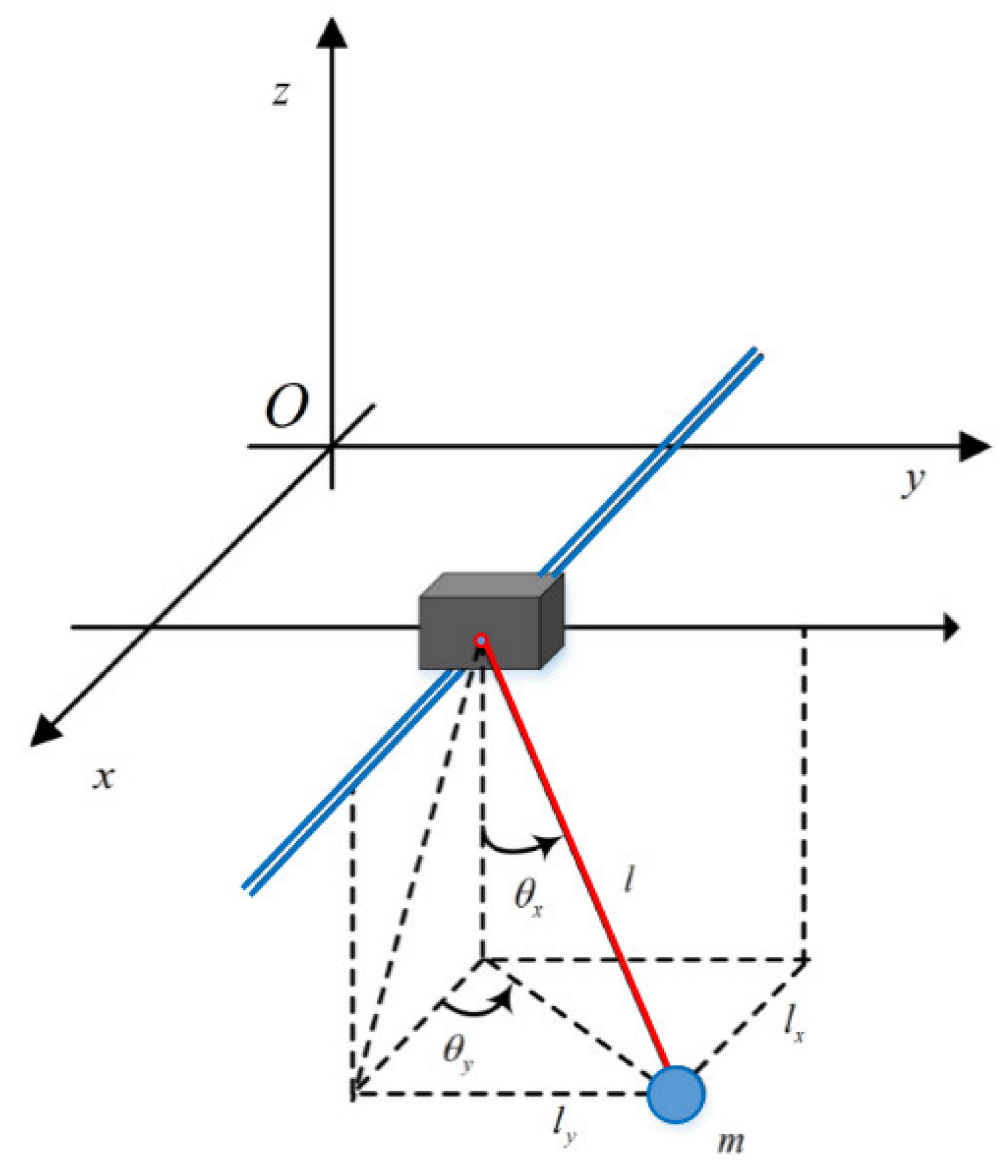

Figure 1. The physical model of a 3-dimensional overhead crane.

If the positions of the trolley on the $x y$ plane of the Cartesian coordinate system are defined by $x$ and $y$, then an overhead crane is a multiple input multiple output (MIMO) system, where the inputs and outputs are $f_{1}=\left[\begin{array}{ll}u_{x} & u_{y}\end{array}\right]^{T} \in R^{2}$ and $q=\left[\begin{array}{llll}x & y & \theta_{x} & \theta_{y}\end{array}\right] \in R^{4}$, respectively. Given its inputs and outputs, the overhead crane evidently has an under-actutated mechanical system. 
Given the aforesaid definitions, the dynamic model of a 3D overhead crane can be presented by the use of Lagrange's equations of motion of second kind, as follows,

$$
\frac{d}{d t}\left(\frac{\partial L(\underline{q}, \dot{q})}{\partial \underline{\dot{q}}}\right)^{T}-\left(\frac{\partial L(\underline{q}, \underline{\dot{q}})}{\partial \underline{q}}\right)^{T}=\underline{u}
$$

where

$$
\begin{aligned}
L= & \frac{1}{2}\left(m_{c}+m\right) \dot{x}^{2}+\frac{1}{2}\left(m_{c}+m+m_{r}\right) \dot{y}^{2}+\frac{1}{2}\left(m l^{2}\right) \dot{\theta}_{x}^{2}+ \\
& \frac{1}{2}\left(m l^{2} \sin ^{2} \theta_{x}\right) \dot{\theta}_{y}^{2}+m l \dot{x} \dot{\theta}_{x} \cos \theta_{x} \cos \theta_{y}-m l \dot{x} \dot{\theta}_{y} \sin \theta_{x} \sin \theta_{y}+ \\
& m l \dot{y} \dot{\theta}_{y} \cos \theta_{x} \sin \theta_{y}+m l \dot{y} \dot{\theta}_{y} \sin \theta_{x} \cos \theta_{y}+m g l \cos \theta_{x}
\end{aligned}
$$

and $\underline{q}=\left[\begin{array}{llll}x & y & \theta_{x} & \theta_{y}\end{array}\right]^{T}$ is the system state vector.

By taking the derivative of (1) with respect to $q$, it yields the dynamic model of the 3D gantry system in the matrix form specified by

$$
M(\underline{q}) \ddot{q}+C(\underline{q}, \underline{\dot{q}}) \dot{q}+D \dot{q}+\underline{g}(\underline{q})=\underline{u}
$$

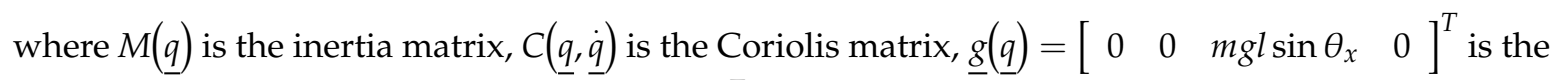
gravitational vector and $\underline{u}=\left[\begin{array}{llll}u_{x} & u_{y} & 0 & 0\end{array}\right]^{T}$ is the control vector. These matrices and vectors are computed by

$$
\begin{gathered}
M=\left[\begin{array}{llll}
m_{11} & m_{12} & m_{13} & m_{14} \\
m_{21} & m_{22} & m_{23} & m_{24} \\
m_{31} & m_{32} & m_{33} & m_{34} \\
m_{41} & m_{42} & m_{43} & m_{44}
\end{array}\right] \quad C=\left[\begin{array}{cccc}
0 & 0 & c_{13} & c_{14} \\
0 & 0 & c_{23} & c_{24} \\
0 & 0 & 0 & c_{34} \\
0 & 0 & c_{43} & c_{44}
\end{array}\right] \quad D=\left[\begin{array}{cccc}
D_{x} & 0 & 0 & 0 \\
0 & D_{y} & 0 & 0 \\
0 & 0 & 0 & 0 \\
0 & 0 & 0 & 0
\end{array}\right] \\
m_{11}=m_{c}+m ; m_{12}=0 ; m_{13}=m l \cos \theta_{x} \cos \theta_{y} ; m_{14}=-m l \sin \theta_{x} \sin \theta_{y} ; \\
m_{21}=0 ; m_{22}=m_{c}+m_{r}+m ; m_{23}=m l \cos \theta_{x} \sin \theta_{y} ; m_{24}=m l \sin \theta_{x} \cos \theta_{y} ; \\
m_{31}=m l \cos \theta_{x} \cos \theta_{y} ; m_{32}=m l \cos \theta_{x} \sin \theta_{y} ; m_{33}=m l^{2} ; m_{34}=0 ; \\
m_{41}=-m l \sin \theta_{x} \sin \theta_{y} ; m_{42}=m l \cos \theta_{x} \sin \theta_{y} ; m_{43}=0 ; m_{44}=m l^{2} \sin ^{2} \theta_{x} \\
c_{13}=-m l \dot{\theta}_{x} \sin \theta_{x} \cos \theta_{y}-m l \dot{\theta}_{y} \cos \theta_{x} \sin \theta_{y} ; c_{14}=-m l \dot{\theta}_{x} \cos \theta_{x} \sin \theta_{y}-m l \dot{\theta}_{y} \sin \theta_{x} \cos \theta_{y} ; \\
c_{23}=-m l \dot{\theta}_{x} \sin \theta_{x} \sin \theta_{y}+m l \dot{\theta}_{y} \cos \theta_{x} \cos \theta_{y} ; c_{24}=m l \dot{\theta}_{x} \cos \theta_{x} \cos \theta_{y}-m l \dot{\theta}_{y} \sin \theta_{x} \sin \theta_{y} ; \\
c_{34}=-m l^{2} \dot{\theta}_{y} \sin \theta_{x} \cos \theta_{y} ; c_{43}=m l^{2} \dot{\theta}_{y} \sin \theta_{x} \cos \theta_{y} ; c_{44}=m l^{2} \dot{\theta}_{x} \sin \theta_{x} \cos \theta_{y}
\end{gathered}
$$

\subsection{A Sliding Mode Controller for a 3D Overhead Crane}

Robustly controlling a 3D overhead crane is to effectively drive both the bridge and trolley onto the predefined paths in $x$-direction and $y$-direction, and efficiently minimize the payload swings $\left(\theta_{x}, \theta_{y}\right)$, given the unknown cargo mass and uncertain matrices $M(q), C(q, \dot{q}), g(q)$. To deal with a system with the high uncertainties of a 3D gantry crane, the SMC approach would provide remarkable robustness in the control performance [30]. If we divide the matrices and vectors in (3) into the submatrice $R^{2 \times 2}$ and the subvector $R^{2 \times 1}$ then (3) can be represented by

$$
\left[\begin{array}{ll}
M_{11} & M_{12} \\
M_{21} & M_{22}
\end{array}\right]\left[\begin{array}{l}
\ddot{q}_{1} \\
\ddot{q}_{2}
\end{array}\right]+\left[\begin{array}{ll}
C_{11} & C_{12} \\
C_{21} & C_{22}
\end{array}\right]\left[\begin{array}{l}
\dot{q}_{1} \\
\dot{q}_{2}
\end{array}\right]+\left[\begin{array}{ll}
D_{11} & D_{12} \\
D_{21} & D_{22}
\end{array}\right]\left[\begin{array}{c}
\dot{q}_{1} \\
\dot{q}_{2} \\
\underline{q}_{2}
\end{array}\right]+\left[\begin{array}{c}
\underline{G}_{1} \\
\underline{G}_{2}
\end{array}\right]=\left[\begin{array}{c}
f \\
\underline{-}_{1} \\
0
\end{array}\right]
$$

where $M_{11}, M_{12}, M_{21}, M_{22} \in R^{2 \times 2}, C_{11}, C_{12}, C_{21}, C_{22} \in R^{2 \times 2}, D_{11}, D_{12}, D_{21}, D_{22} \in R^{2 \times 2}$, and $G_{1}, G_{2} \in R^{2 \times 1}$. 
Note that

$$
\begin{gathered}
M=\left[\begin{array}{ll}
M_{11} & M_{12} \\
M_{21} & M_{22}
\end{array}\right], \\
M_{11}=\left[\begin{array}{ll}
m_{11} & m_{12} \\
m_{21} & m_{22}
\end{array}\right], M_{12}=\left[\begin{array}{ll}
m_{13} & m_{14} \\
m_{23} & m_{24}
\end{array}\right], M_{21}=\left[\begin{array}{ll}
m_{31} & m_{32} \\
m_{41} & m_{42}
\end{array}\right], M_{22}=\left[\begin{array}{ll}
m_{33} & m_{34} \\
m_{43} & m_{44}
\end{array}\right] \\
C=\left[\begin{array}{ll}
C_{11} & C_{12} \\
C_{21} & C_{22}
\end{array}\right], C_{11}=\left[\begin{array}{ll}
0 & 0 \\
0 & 0
\end{array}\right], C_{12}=\left[\begin{array}{ll}
c_{13} & c_{14} \\
c_{23} & c_{24}
\end{array}\right], C_{21}=\left[\begin{array}{ll}
0 & 0 \\
0 & 0
\end{array}\right], C_{22}=\left[\begin{array}{cc}
0 & c_{34} \\
c_{43} & c_{44}
\end{array}\right] \\
D=\left[\begin{array}{ll}
D_{11} & D_{12} \\
D_{21} & D_{22}
\end{array}\right], D_{11}=\left[\begin{array}{cc}
D_{x} & 0 \\
0 & D_{y}
\end{array}\right], D_{12}=\left[\begin{array}{ll}
0 & 0 \\
0 & 0
\end{array}\right], D_{21}=\left[\begin{array}{ll}
0 & 0 \\
0 & 0
\end{array}\right], D_{22}=\left[\begin{array}{ll}
0 & 0 \\
0 & 0
\end{array}\right]
\end{gathered}
$$

Thus, we can now present (4) into the two matrix equations as follows,

$$
\begin{gathered}
M_{11} \ddot{\ddot{q}}_{1}+M_{12} \ddot{q}_{2}+C_{11} \dot{q}_{1}+C_{12} \dot{q}_{2}+D_{11} \dot{q}_{1}+\underline{G}_{1}=\underline{f}_{1} \\
M_{21} \ddot{\ddot{q}}_{1}+M_{22} \ddot{q}_{2}+C_{21} \dot{q}_{1}+C_{22} \underline{q}_{2}+\underline{G}_{2}=0
\end{gathered}
$$

Since $M_{22}$ is positive, the equation (6) can be rewritten by

$$
\underline{\ddot{q}}_{2}=-\left(M_{22}\right)^{-1}\left(M_{21} \ddot{\ddot{q}}_{1}+C_{21} \underline{\dot{q}}_{1}+C_{22} \underline{\dot{q}}_{2}+G_{2}\right)
$$

From (5) and (7), it yields

$$
M_{11} \ddot{q}_{1}+M_{12}\left(-\left(M_{22}\right)^{-1}\left(\begin{array}{c}
M_{21} \ddot{q}_{1}+C_{21} \dot{q}_{1} \\
+C_{22} \underline{q}_{2}+G_{2}
\end{array}\right)\right)+C_{11} \dot{q}_{1}+C_{12} \dot{q}_{2}+D_{11} \dot{q}_{1}+\underline{G}_{1}=\underline{f}_{1}
$$

or

$$
\bar{M}_{1} \ddot{q}_{1}+\bar{C}_{1} \dot{q}_{1}+\bar{C}_{2} \dot{q}_{2}+\bar{D} \dot{q}_{1}+\bar{G}_{1}=\underline{f}_{1}
$$

where

$$
\begin{gathered}
\bar{M}_{1}=M_{11}-M_{12}\left(M_{22}\right)^{-1} M_{21}=\left[\begin{array}{cc}
m_{c}+m \sin ^{2} \theta_{x} \cos ^{2} \theta_{y} & -m \cos \theta_{y} \sin \theta_{y}\left(1+\cos ^{2} \theta_{x}\right) \\
-m \cos \theta_{y} \sin \theta_{y}\left(1+\cos ^{2} \theta_{x}\right) & m_{c}+m_{r}+m \sin ^{2} \theta_{x} \sin ^{2} \theta_{y}
\end{array}\right], \\
\bar{C}_{1}=C_{11}-M_{12}\left(M_{22}\right)^{-1} C_{21}=\left[\begin{array}{ll}
0 & 0 \\
0 & 0
\end{array}\right], \\
\bar{C}_{2}=C_{12}-M_{12}\left(M_{22}\right)_{-1} C_{22} \\
=\left[\begin{array}{ll}
-m l \dot{\theta}_{y} \sin \theta_{y} \cos \theta_{y} & m l \dot{\theta}_{y} \sin \theta_{x} \cos \theta_{x} \cos ^{2} \theta_{y}-m l \dot{\theta}_{x} \sin \theta_{y} \cos \theta_{y} \\
-m l \dot{\theta}_{y} \cos ^{2} \theta_{y} & m l \dot{\theta}_{y} \sin \theta_{x} \cos \theta_{x} \sin \theta_{y} \cos \theta_{y}-m i \dot{\theta}_{x} \cos ^{2} \theta_{y}
\end{array}\right] \\
\bar{G}_{1}=G_{1}-M_{12}\left(M_{22}\right)^{-1} \underline{G}_{2}=\left[\begin{array}{c}
-m g \cos \theta_{x} \cos \theta_{y} \sin \theta_{x} \\
-m g \cos \theta_{x} \sin \theta_{y} \sin \theta_{x}
\end{array}\right], \\
\bar{D}=D_{11}=\left[\begin{array}{cc}
D_{x} & 0 \\
0 & D_{y}
\end{array}\right] .
\end{gathered}
$$


If the sliding surface is defined by

$$
s=\dot{q}_{1}+\lambda\left(q_{1}-q_{1 d}\right)+\alpha q_{2}
$$

where $\underline{q}_{1 d}$ is the desired position of the trolley that is constant in a duty cycle, then $\lambda$ and $\alpha$ are positive diagonal matrices. Therefore, the derivative of the sliding surface is

$$
\underline{\dot{s}}=\ddot{q}_{1}+\lambda \underline{q}_{1}+\alpha \dot{q}_{2}=\left(\bar{M}_{1}\right)^{-1}\left(\underline{f}_{1}-\bar{C}_{2} \dot{q}_{2}-\bar{G}_{1}-\bar{D} \underline{q}_{1}\right)+\lambda \underline{q}_{1}+\alpha \dot{q}_{2}
$$

If we choose the control law $\dot{s}=-\bar{M}_{1}\left(K_{1} \operatorname{sgn}(s)+K_{2} s\right)$ where $K_{1}, K_{2} \in R^{2 \times 2}$ are positive diagonal matrices and $\operatorname{sgn}()$ is the sign function, then the control input that can maintain the stability of the sliding surface is given by

$$
\underline{f}_{1}=\bar{C}_{2} \dot{q}_{2}+\bar{G}_{1}+\bar{D} \underline{q}_{1}-\bar{M}_{1}^{-1}\left(\lambda \dot{q}_{1}+\alpha \dot{q}_{2}+K_{1} \operatorname{sgn}(s)+K_{2} s\right)
$$

In practice, to reduce the chattering phenomenon, the saturation function is used instead of the sign function as follows,

$$
\tau_{s}= \begin{cases}\tau_{\max } & \text { if } \tau>\tau_{\max } \\ \tau & \text { if } \tau_{\min }<\tau<\tau_{\max } \\ \tau_{\min } & \text { if } \tau<\tau_{\min }\end{cases}
$$

where $\tau_{S}$ and $\tau$ are the actuator output and control input, respectively. $\tau_{\max }$ and $\tau_{\min }$ are the upper and lower bounds of $\tau$. For more information about the saturation function in designing a SMC scheme, interested readers are referred to our previous works [30].

\section{An Adaptive Sliding Mode Controller for Uncertain Payload-Mass 3D Overhead Cranes}

Though the SMC controller designed in the previous section provides the robustness and stability of a crane system with uncertainties and nonlinearities, it does not have a mechanism to estimate the uncertain and unknown system parameters, which prevents it from practicality in real-life control applications, especially a 3D overheard crane. To this end, we propose to employ the radial basis function neural network (RBFNN) to adaptively estimate the unknown and uncertain parameters for the designed SMC controller.

\subsection{An Adaptive Controller}

In estimation of the nonlinear characteristics of a system, a two-layer RBFNN is frequently utilized, where the input layers are the radial neurons while the output layers are the linear neurons. In this work, we define the inputs for the RBFNN as $\underline{q}=\left[\begin{array}{llll}x & y & \theta_{x} & \theta_{y}\end{array}\right]^{T} \in R^{4}$ and $\underline{\dot{q}}=\left[\begin{array}{cccc}\dot{x} & \dot{y} & \dot{\theta}_{x} & \dot{\theta}_{y}\end{array}\right]^{T} \in R^{4}$ for the position vector and the velocity vector, respectively. And $\underline{\hat{\delta}}=\left[\begin{array}{ll}\hat{\delta}_{1} & \hat{\delta}_{2}\end{array}\right]^{T} \in R^{2}$ is defined as the output of the RBFNN. Inside the RBFNN, we denote $W \in R^{l \times 2}$ as the ideal weight matrix, where $l$ is the number of the neurons in a hidden layer. Moreover, the activation functions are summarized by $\underline{h}=\left[\begin{array}{llll}h_{1} & h_{2} & \ldots & h_{l}\end{array}\right]^{T} \in R^{l}$, where their component is computed by

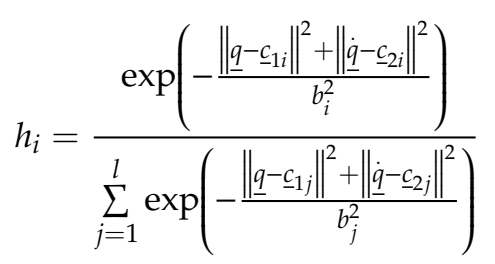

The structure of a RBFNN is visually demonstrated in Figure 2. 


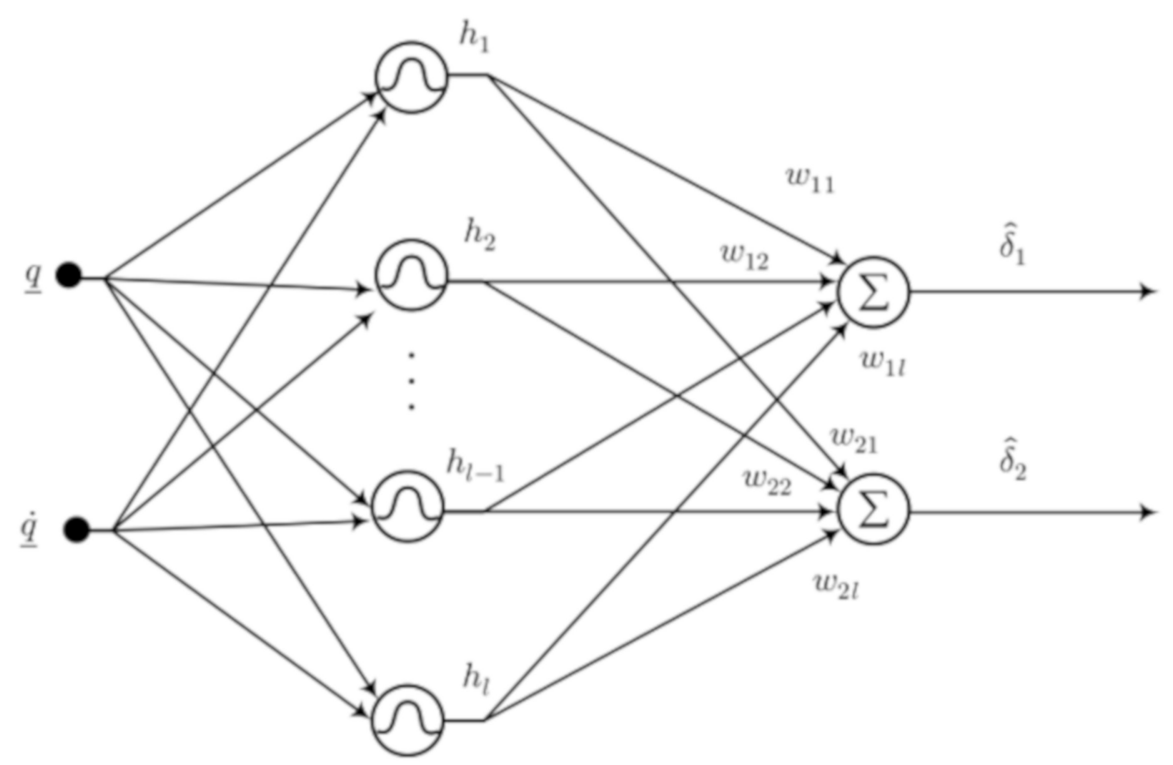

Figure 2. A radial basis function neural network.

We define the uncertainty as follows,

$$
\underline{\delta}=\frac{1}{m}\left(\bar{C}_{2} \dot{q}_{2}+\bar{G}_{1}\right)
$$

Since $\bar{C}_{2}$ and $\bar{G}_{1}$ are uncertain and unknown, we propose to employ the RBFNN to estimate them. Ideally, the output of the RBFNN is presented by $\underline{\delta}=W^{T} \underline{h}+\underline{\varepsilon}$ where $\underline{\varepsilon}$ is the infinitesimal error. If $\hat{W}$ is the estimation of $W$, the RBFNN output is $\underline{\hat{\delta}}=\hat{W}^{T} \underline{h}$. Then $\bar{W}=W-\hat{\hat{W}}$ is the error of the weight matrix.

In fact, the payload mass $m$ is unknown and varies over time. If we assume $\hat{m}$ as the approximation of $m$, which is adaptively estimated and updated online, the approximate matrices in (9) can be given by

$$
\begin{gathered}
\hat{\bar{M}}_{1}=\hat{m}\left[\begin{array}{cc}
\frac{m_{c}}{\hat{m}}+\sin ^{2} \theta_{x} \cos ^{2} \theta_{y} & -\cos \theta_{y} \sin \theta_{y}\left(1+\cos ^{2} \theta_{x}\right) \\
-\cos \theta_{y} \sin \theta_{y}\left(1+\cos ^{2} \theta_{x}\right) & \frac{m_{c}+m_{r}}{\hat{m}}+\sin ^{2} \theta_{x} \sin ^{2} \theta_{y}
\end{array}\right] \\
\hat{\bar{C}}_{2}=\hat{m}\left[\begin{array}{cc}
-i \dot{\theta}_{y} \sin \theta_{y} \cos \theta_{y} & i \dot{\theta}_{y} \sin \theta_{x} \cos \theta_{x} \cos ^{2} \theta_{y}-i \dot{\theta}_{x} \sin \theta_{y} \cos \theta_{y} \\
-l \dot{\theta}_{y} \cos ^{2} \theta_{y} & i \dot{\theta}_{y} \sin \theta_{x} \cos \theta_{x} \sin \theta_{y} \cos \theta_{y}-l \dot{\theta}_{x} \cos ^{2} \theta_{y}
\end{array}\right] \\
\hat{\bar{G}}_{1}=\hat{m}\left[\begin{array}{c}
-g \cos \theta_{x} \cos \theta_{y} \sin \theta_{x} \\
-g \cos \theta_{x} \sin \theta_{y} \sin \theta_{x}
\end{array}\right]
\end{gathered}
$$

As a result, the control input (12) can be approximately calculated by

$$
\underline{f}_{1}=\bar{D} \underline{q}_{1}+\hat{m} \underline{\hat{\delta}}-\hat{\bar{M}}_{1}^{-1}\left(\lambda \underline{q}_{1}+\alpha \dot{q}_{2}+K_{1} \operatorname{sgn}(\underline{s})+K_{2} \underline{s}\right)
$$

We now define a Lyapunov function as follows,

$$
V=\frac{1}{2} \underline{s}^{T} \bar{M}_{1} \underline{s}+\frac{1}{2} \gamma \widetilde{m}^{2}+\frac{1}{2} \operatorname{tr}\left(m \widetilde{W}^{T} F^{-1} \widetilde{W}\right)
$$

where $\widetilde{m}=m-\hat{m}, \gamma$ is a positive number, and $F \in R^{l \times l}$ is a positive diagonal matrix. Then the derivative of the Lyapunov function, with respect to time, is computed by

$$
\dot{V}=\frac{1}{2} s^{T} \frac{\dot{M}}{s}+\underline{s}^{T} \bar{M} \underline{s}-\gamma \widetilde{m} \dot{\hat{m}}-\operatorname{tr}\left(m \widetilde{W}^{T} F^{-1} \dot{\hat{W}}\right)
$$


By utilizing the control input (14), the derivative of the Lyapunov function can be rewritten as follows,

$$
\dot{V}=\underline{s}^{T}\left(\begin{array}{c}
\widetilde{\bar{M}}_{1}\left(-\lambda \dot{q}-\alpha \dot{q}_{1}-K_{1} \operatorname{sgn}(\underline{s})-K_{2} \underline{s}\right) \\
-\widetilde{m} \underline{\hat{\delta}}-m \underline{\widetilde{\delta}}-\bar{M}_{1} K_{1} \operatorname{sgn}(\underline{s})-\bar{M}_{1} K_{2} \underline{s}
\end{array}\right)+\frac{1}{2} \underline{s}^{T} \dot{\bar{M}} \underline{s}-\gamma \widetilde{m} \dot{\hat{m}}-\operatorname{tr}\left(m \widetilde{W}^{T} F^{-1} \dot{\hat{W}}\right)
$$

where $\widetilde{\bar{M}}_{1}=\bar{M}_{1}-\hat{\bar{M}}_{1}=\widetilde{m} \Lambda$ and

$$
\Lambda=\left[\begin{array}{cc}
\sin ^{2} \theta_{x} \cos ^{2} \theta_{y} & -\cos \theta_{y} \sin \theta_{y}\left(1+\cos ^{2} \theta_{x}\right) \\
-\cos \theta_{y} \sin \theta_{y}\left(1+\cos ^{2} \theta_{x}\right) & \sin ^{2} \theta_{x} \sin ^{2} \theta_{y}
\end{array}\right] .
$$

Therefore,

$$
\begin{aligned}
\dot{V}= & s^{T}\left(\frac{1}{2} \dot{\bar{M}}_{1}-\bar{M}_{1} K_{2}\right) s-\underline{s}^{T} \bar{M}_{1} K_{1} \operatorname{sgn}(\underline{s})-s^{T} m \varepsilon \\
& +\widetilde{m}\left(s^{T} \Lambda\left(\begin{array}{c}
-\lambda \dot{q}_{1}-\alpha \dot{q}_{2} \\
-K_{1} \operatorname{sgn}(s)-K_{2} s
\end{array}\right)-s^{T} \hat{\delta}-\gamma \dot{\hat{m}}\right)+\operatorname{tr}\left(-m \widetilde{W}^{T}\left(h s^{T}+F^{-1} \dot{\hat{W}}\right)\right)
\end{aligned}
$$

If the adaptation mechanisms for the payload mass and weight matrix are chosen by

$$
\begin{gathered}
\dot{\hat{m}}=\gamma^{-1}\left(s^{T} \Lambda\left(-\lambda \dot{q}_{1}-\alpha \dot{q}_{2}-K_{1} \operatorname{sgn}(s)-K_{2} s\right)-s^{T} \hat{\delta}\right), \\
\dot{\hat{W}}=-F h s^{T},
\end{gathered}
$$

then

$$
\begin{aligned}
\dot{V}= & -\underline{s}^{T}\left(\bar{M}_{1} K_{2}-\frac{1}{2} \dot{\bar{M}}_{1}\right) \underline{s}-\underline{s}^{T} \bar{M}_{1} K_{1} \operatorname{sgn}(\underline{s})-\underline{s}^{T} m \underline{\varepsilon} \\
& \leq-\underline{s}^{T}\left(\bar{M}_{1} K_{2}-\frac{1}{2} \dot{\bar{M}}_{1}\right) \underline{s}-\|\underline{s}\|\left(\lambda_{\min }-m\|\varepsilon\|\right)
\end{aligned}
$$

where $\lambda_{\min }$ is the smallest value of the matrix $\bar{M}_{1} K_{1}$.

\subsection{An Adaptive Controller}

The effectiveness of the proposed adaptive uncertain payload mass controller for a 3D overhead crane can be mathematically demonstrated by the stability of the closed-loop control system in the following theorem:

Theorem 1: Given the uncertainty component $\underline{\delta}$ approximated by the RBFNN with the adaptation mechanisms (19) and (20) to update online the unknown and uncertain payload mass and RBFNN weight matrix, the closed-loop control system (4) employing the designed adaptive controller (14) will be asymptotically stable if $\lambda_{\min }-m\|\varepsilon\|>0$ is the smallest value of the matrix $\bar{M}_{1} K_{1}$ and $\bar{M}_{1} K_{2}-\frac{1}{2} \dot{\bar{M}}_{1}$ is the positive define matrix.

Proof : Let us consider the Lyapunov function candidate

$$
V=\frac{1}{2} \underline{s}^{T} \underline{\underline{S}}+\operatorname{tr}\left(\widetilde{W}^{T} F^{-1} \widetilde{W}\right),
$$

from (14), (19), (20) and $\lambda_{\min }-m\|\varepsilon\|>0$ one has

$$
\dot{V} \leq-\underline{s}^{T}\left(\bar{M}_{1} K_{2}-\frac{1}{2} \dot{\bar{M}}_{1}\right) \underline{s}-\|\underline{s}\|\left(\lambda_{\min }-m\|\varepsilon\|\right) \leq 0 .
$$


In other words, the closed-loop control system is asymptotically stable. Nonetheless, since $\bar{M}_{1} K_{1}$ is the positive diagonal matrix, to guarantee $\lambda_{\min }>m\|\varepsilon\|, K_{1}$ must be large enough. On the other hand, as $\bar{M}_{1} K_{2}-\frac{1}{2} \dot{\bar{M}}_{1}$ is the symmetric matrix, it can be represented by

$$
\bar{M}_{1} K_{2}-\frac{1}{2} \dot{\bar{M}}_{1}=\left[\begin{array}{ll}
a & b \\
b & c
\end{array}\right]
$$

where

$$
a=m_{11} k_{11}-\frac{1}{2} \dot{m}_{11} ; b=-\frac{1}{2} \dot{m}_{12} ; c=m_{22} k_{22}-\frac{1}{2} \dot{m}_{22}
$$

and $k_{11}, k_{22}$ are the elements of the diagonal matrix $K_{2}$.

Furthermore, if $\bar{M}_{1} K_{2}-\frac{1}{2} \dot{\bar{M}} 1$ is positive, the polynomial $\operatorname{det}\left(\bar{M}_{1} K_{2}-\frac{1}{2} \dot{\bar{M}}_{1}-\lambda I\right)$ must have two positive roots. That is,

$$
a, c>0 ; a c-b^{2}>0 .
$$

Combining (25) and (26), it yields

$$
\begin{gathered}
m_{11} k_{11}-\frac{1}{2} \dot{m}_{11}>0 ; \quad m_{22} k_{22}-\frac{1}{2} \dot{m}_{22}>0 \\
\left(m_{11} k_{11}-\frac{1}{2} \dot{m}_{11}\right)\left(m_{22} k_{22}-\frac{1}{2} \dot{m}_{22}\right)-\frac{1}{4} \dot{m}_{12}{ }^{2}>0
\end{gathered}
$$

As $m_{11}, m_{22}$ are positive numbers, if $k_{11}, k_{22}$ are large enough, the inequations (27) are held. In other words, there always exists a positive diagonal matrix $K_{2}$ so that $\bar{M}_{1} K_{2}-\frac{1}{2} \dot{\bar{M}}_{1}$ is positively defined.

\section{Simulations and Experimental Results}

To demonstrate the effectiveness of our proposed algorithm, adaptive neural network-based sliding mode controller (ANNSMC), in controlling a 3D overhead crane under the payload mass uncertainty, we conducted the experiments in the synthetic simulation environment and the obtained results are presented in this section. It is noted that the control performance is measured by how efficiently the bridge, trolley and hoist of the 3D gantry crane respond to a control action and whether the trolley can be driven to the desired position with the minimal sway angles.

\begin{tabular}{|c|c|}
\hline Parameters & Values \\
\hline \multicolumn{2}{|c|}{ Crane's dynamic model properties } \\
\hline$m_{r}$ & $50(\mathrm{~kg})$ \\
\hline$m_{c}$ & $30(\mathrm{~kg})$ \\
\hline$l$ & $1(m)$ \\
\hline$g$ & $9.81\left(\mathrm{~m} / \mathrm{s}^{2}\right)$ \\
\hline \multicolumn{2}{|c|}{ Adaptive controller characteristics } \\
\hline$K_{1}$ & $\operatorname{diag}\left(\begin{array}{ll}100 & 100\end{array}\right)$ \\
\hline$K_{2}$ & $\operatorname{diag}\left(\begin{array}{ll}50 & 50\end{array}\right)$ \\
\hline$\alpha$ & $\operatorname{diag}\left(\begin{array}{ll}-5 & -5\end{array}\right)$ \\
\hline$\lambda$ & $\operatorname{diag}\left(\begin{array}{ll}0.5 & 0.5\end{array}\right)$ \\
\hline$\gamma$ & 1 \\
\hline
\end{tabular}

In the experiments, it was assumed that the masses of both the trolley and bridge were known. The length of the hoist cable was also known, although it was supposed to be massless. Some constant parameters of the proposed adaptive controller were given. Those parameters are summarized in Table 1.

Table 1. The parameters of a 3D overhead crane and its controller characteristics. 
It is important to note that in our experiments we assumed that the payload was unknown and that the controller had to adaptively estimate it.

In order to estimate the uncertain and unknown parameters, we designed a two-layer RBFNN with ten neural components. All the weights of the RBFNN were initialized by 0.1 . The simulation block diagram in our experiments is demonstrated in Figure 3.

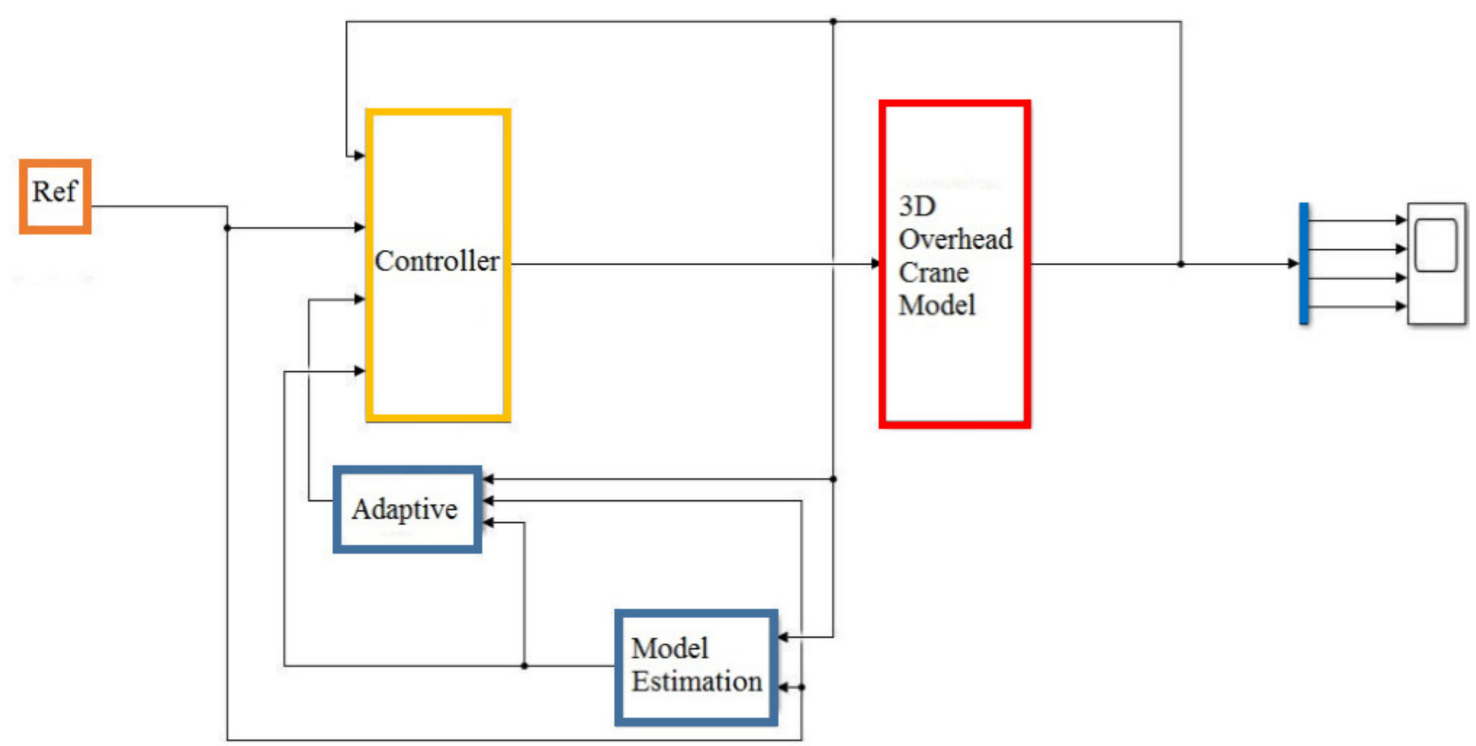

Figure 3. The simulation block diagram.

In order to verify the consistency of the control performance in the 3D crane system given the uncertainty of the payload mass, we set the two different cargo mass values at $10 \mathrm{~kg}$ and $20 \mathrm{~kg}$, respectively, in the two different transporting operations, which were unknown to the controller. It can be seen that though the payload mass was unknown, in both of the transporting operation scenarios, the control force signals in each direction as demonstrated in Figure 4 were quite similar. That is, the control input was mostly independent from the payload mass.

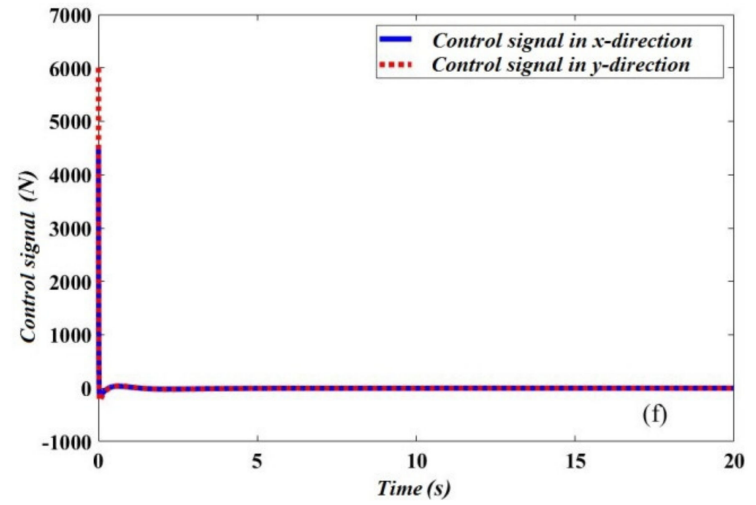

(a) When the payload mass is $10 \mathrm{~kg}$

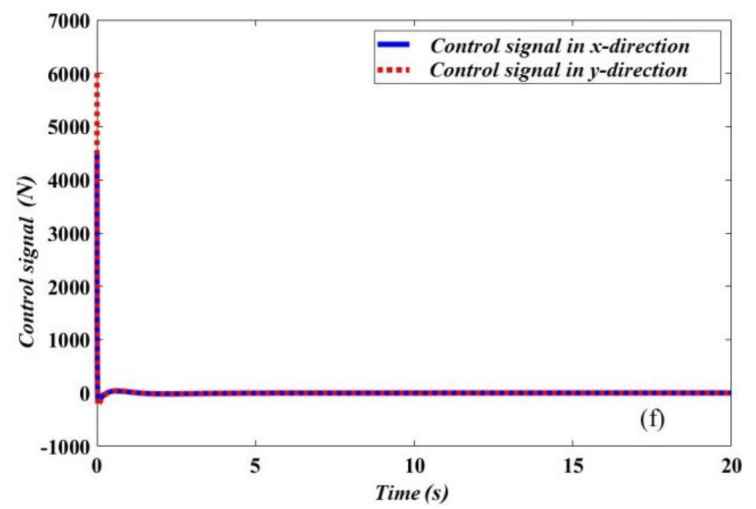

(b) When the payload mass is $20 \mathrm{~kg}$

Figure 4. The control force signals.

Now let us examine the outputs of the 3D overhead crane system in the two transporting operation scenarios conducted. This under-actuated mechanical system has four outputs, including the two coordinates of the trolley positions as shown in Figures 5 and 6, and the two sway angles in each experiment as demonstrated in Figures 7 and 8, for the simulations of the payload masses of $10 \mathrm{~kg}$ and $20 \mathrm{~kg}$, respectively. 
From Figures 5 and 6, it can be clearly seen that after about $5 \mathrm{~s}$, in both scenarios, the trolley reached within $99 \%$ of the desired position. More importantly, in both of the illustrated examples, the responses of the trolley were highly comparable, although the crane transported the different payload masses. The similarity in the trolley responses was a result of the similar control inputs, which were adaptively computed by the RBFNN and adaptation mechanisms, as demonstrated in Figure 4.
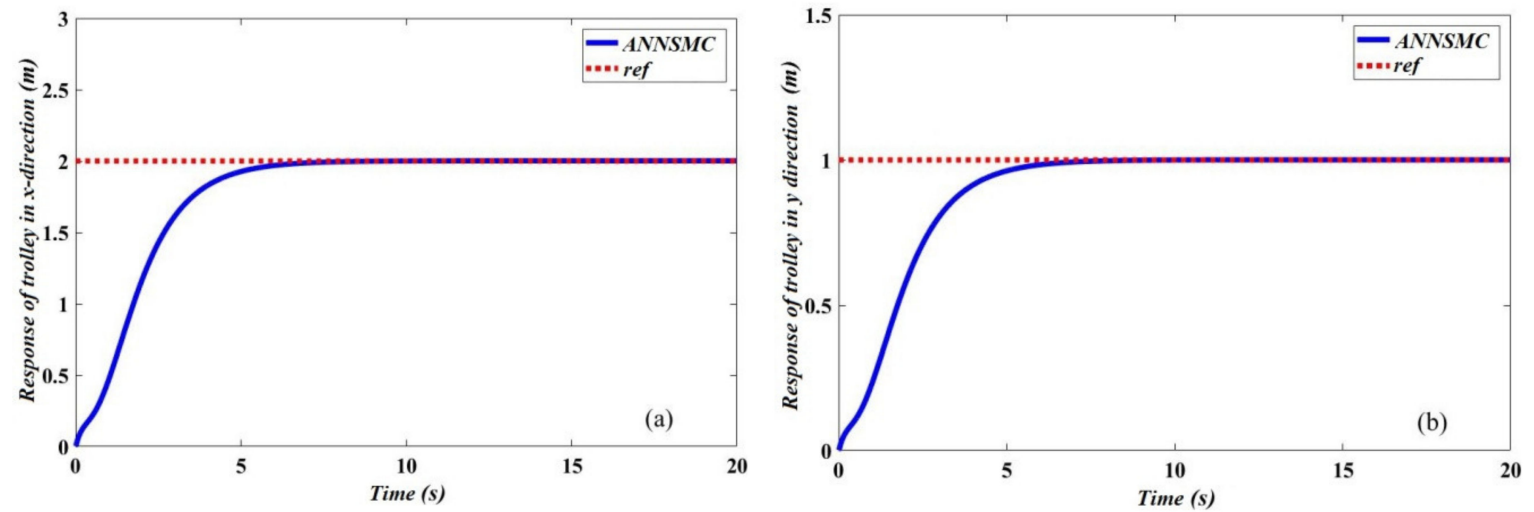

Figure 5. The trolley position when the payload mass is $10 \mathrm{~kg}$.
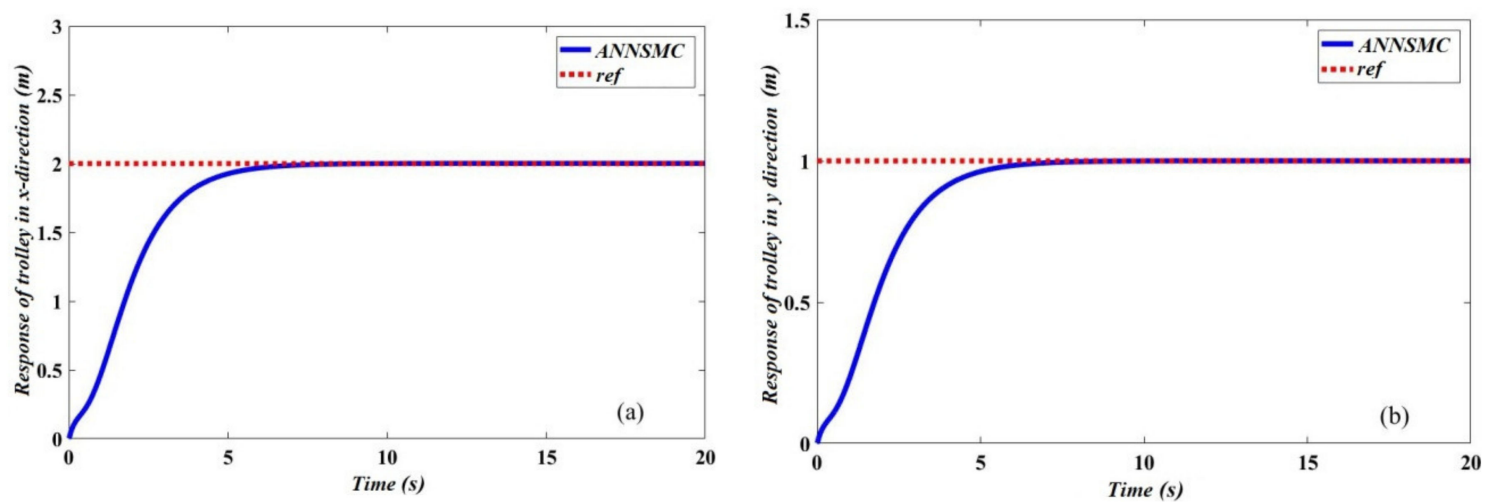

Figure 6. The trolley position when the payload mass is $20 \mathrm{~kg}$.
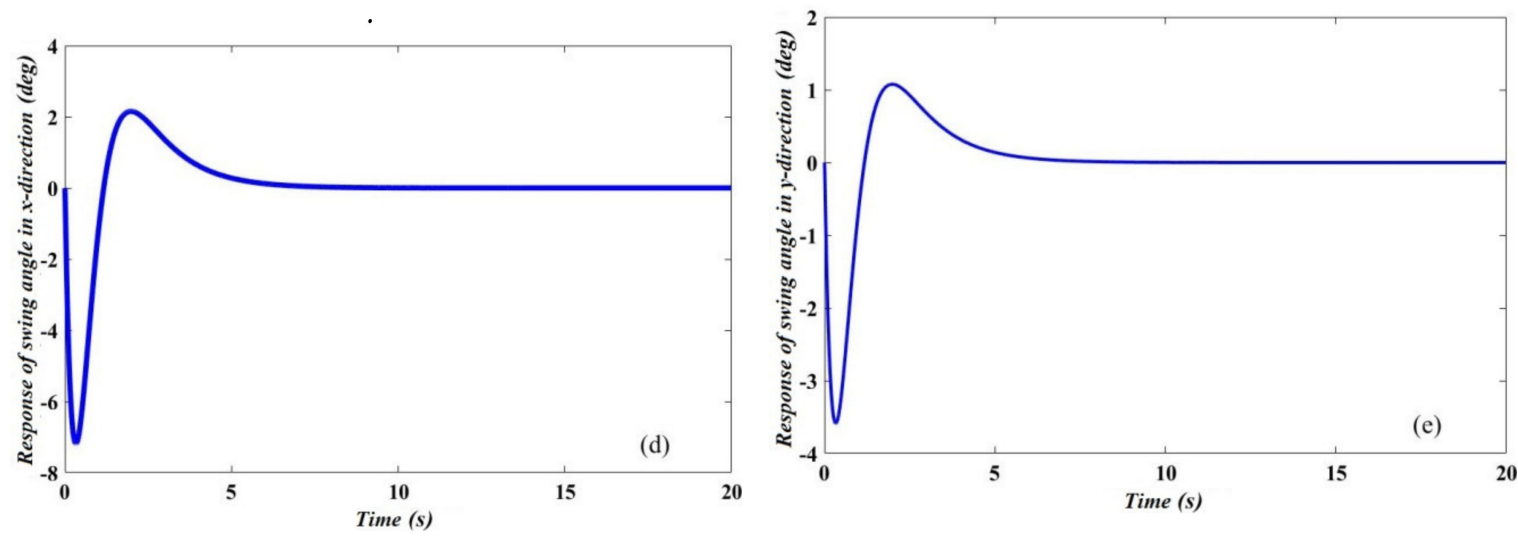

Figure 7. The swing angles when the payload mass is $10 \mathrm{~kg}$. 

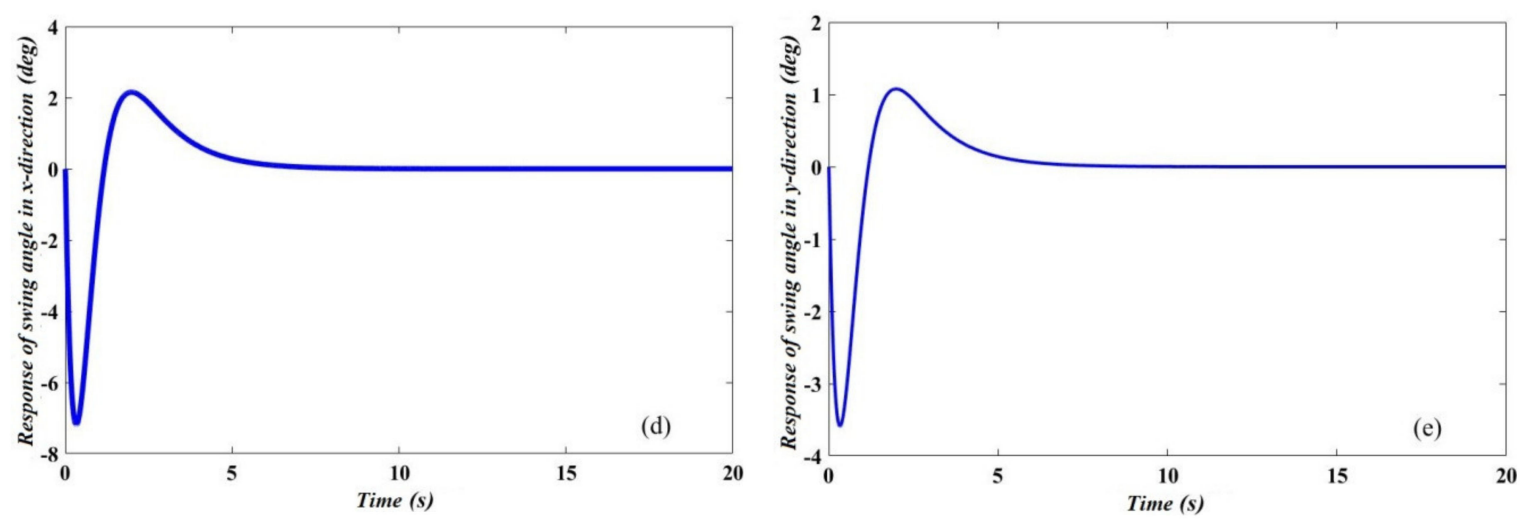

Figure 8. The swing angles when the payload mass is $20 \mathrm{~kg}$.

In addition to the positions of the trolley, the two other outputs of the gantry crane included the payload swing angles, as illustrated in Figure 7, and the consistency of the control performance, as shown in Figure 8, where the maximum sway angles in the two presented directions in any scenario were about 8 and 4 degrees. These characteristics of the proposed control algorithm are practically crucial in terms of maintaining the reliability, stability and robustness of the closed-loop control system. In other words, the designed controller is highly applicable.

\section{Conclusions}

This paper has proposed an adaptive control scheme based on the SMC structure for the 3D uncertain overhead crane under the payload mass uncertainty. Moreover, the unknown and uncertain parameters of the system dynamics are proposed to be adaptively estimated by the use of the RBFNN. By proposing the adaptation mechanisms derived from the Lyapunov theory to approximate the unknown payload mass and weight matrix, the paper mathematically proves the stability of the closed-loop control system. The results obtained by implementing the proposed algorithm in the simulations have shown that the trolley could reach the reference position within $5 \mathrm{~s}$, and the maximum sway angles in both directions are 8 and 4 degrees, respectively. More importantly, the results were not dependent on the payload, which has demonstrated the consistency of the control performance, although the crane transported the payloads with the different masses. In future works, we will verify the method in the real-life systems.

Author Contributions: All the authors conducted the research and discussed to find the solution of the problem. U.T.T.H., L.N. and H.X.L. conducted analysis and wrote Sections 2 and 3. N.H.T., H.V.P. and L.N. conducted the simulations and wrote the rest of the paper. All authors have read and agreed to the published version of the manuscript.

Funding: This research received no external funding.

Conflicts of Interest: The authors declare no conflict of interest.

\section{References}

1. Butler, H.; Honderd, G.; Van Amerongen, J. Model reference adaptive control of a gantry crane scale model. IEEE Control Syst. Mag. 1991, 11, 57-62. [CrossRef]

2. Yoon, J.; Nation, S.; Singhose, W.; Vaughan, J.E. Control of crane payloads that bounce during hoisting. IEEE Trans. Control Syst. Technol. 2014, 22, 1233-1238. [CrossRef]

3. Lee, H.H. Motion planning for three-dimensional overhead cranes with high-speed load hoisting. Int. J. Control 2005, 78, 875-886. [CrossRef]

4. Sun, N.; Yang, T.; Chen, H.; Fang, Y.C.; Qian, Y.Z. Adaptive anti-swing and positioning control for 4-DOF rotary cranes subject to uncertain/unknown parameters with hardware experiments. IEEE Trans. Syst. Man Cybern. Syst. 2019, 49, 1309-1321. [CrossRef] 
5. Sun, N.; Yang, T.; Fang, Y.C.; Wu, Y.M.; Chen, H. Transportation control of double-pendulum cranes with a nonlinear quasi-PID scheme: Design and experiments. IEEE Trans. Syst. Man Cybern. Syst. 2019, 49, 1408-1418. [CrossRef]

6. Saeidi, H.; Naraghi, M.; Raie, A.A. A neural network selftuner based on input shapers behavior for anti-sway system of gantry cranes. J. Vib. Control 2013, 19, 1936-1949. [CrossRef]

7. Omar, H.M. Control of Gantry and Tower Cranes. Ph. D. Thesis, Virginia Polytechnic Institute and State University, Blacksburg, VA, USA, 2003.

8. Omar, H.M.; Nayfeh, A.H. Anti-swing control of gantry and tower cranes using fuzzy and time-delayed feedback with friction compensation. Shock Vib. 2005, 12, 73-89. [CrossRef]

9. Fang, Y.; Dixon, W.E.; Dawson, D.M.; Zergeroglu, E. Nonlinear coupling control laws for an underactuated overhead crane system. IEEE/ASME Trans. Mechatron. 2003, 8, 418-423. [CrossRef]

10. Yu, J.; Lewis, F.L.; Huang, T. Nonlinear feedback control of a gantry crane. In Proceedings of the IEEE American Control Conference, Seattle, WA, USA, 21-23 June 1995; pp. 4310-4315. [CrossRef]

11. Park, H.; Chwa, D.; Hong, K.S. A feedback linearization control of container cranes: Varying rope length. Int. J. Control Autom. Syst. 2007, 5, 379-387.

12. Le, T.A.; Lee, S.G.; Dang, V.H.; Moon, S.; Kim, B.S. Partial feedback linearization control of a three-dimensional overhead crane. Int. J. Control Autom. Syst. 2013, 11, 718-727. [CrossRef]

13. Le, T.A.; Kim, G.H.; Kim, M.Y.; Lee, S.G. Partial feedback linearization control of overhead cranes with varying cable lengths. Int. J. Precis. Eng. Manuf. 2012, 13, 501-507. [CrossRef]

14. Le, H.; Nguyen, L.; Thiyagarajan, K.; Pham, H. A Dynamic Surface Controller based on Adaptive Neural Network for Dual Arm Robots. In Proceedings of the International IEEE Conference on Industrial Electronics and Applications, Kristiansand, Norway, 21-25 June 2020. Accepted.

15. Pham, D.T.; Nguyen, T.V.; Le, H.X.; Nguyen, L.; Thai, N.H.; Phan, T.A.; Pham, H.T.; Duong, A.H.; Bui, L.T. Adaptive neural network based dynamic surface control for uncertain dual arm robots. Int. J. Dynam. Control 2019. [CrossRef]

16. Van Nguyen, T.; Thai, N.H.; Pham, H.T.; Phan, T.A.; Nguyen, L.; Le, H.X.; Nguyen, H.D. Adaptive Neural Network-Based Backstepping Sliding Mode Control Approach for Dual-Arm Robots. J. Control Autom. Electr. Syst. 2019, 30, 512-521. [CrossRef]

17. Shyu, K.K.; Jen, C.L.; Shang, L.J. Design of sliding-mode controller for anti-swing control of overhead cranes. In Proceedings of the 31st Annual Conference of IEEE Industrial Electronics Society, Raleigh, NC, USA, 6-10 November 2005; pp. 147-152. [CrossRef]

18. Qian, D.W.; Yi, J.Q.; Zhao, D.B. Control of overhead crane systems by combining sliding mode with fuzzy regulator. IFAC Proc. Vol. 2011, 44, 9320-9325. [CrossRef]

19. Le, T.A.; Kim, J.J.; Lee, S.G.; Lim, T.G.; Luong, N.C. Second-order sliding mode control of a 3D overhead crane with uncertain system parameters. Int. J. Precis. Eng. Manuf. 2014, 15, 811-819. [CrossRef]

20. Ngo, Q.H.; Hong, K.S. Sliding-mode antisway control of an offshore container crane. IEEE/ASME Trans. Mechatron. 2012, 17, 201-209. [CrossRef]

21. Bartolini, G.; Pisano, A.; Usai, E. Second-order sliding mode control of container cranes. Automatica 2002, 38, 1783-1790. [CrossRef]

22. Mahjoub, S.; Mnif, F.; Derbel, N. Second-order sliding mode approaches for the control of a class of underactuated systems. Int. J. Autom. Comput. 2015, 12, 134-141. [CrossRef]

23. Xu, W.M.; Zheng, X.; Liu, Y.Q.; Zhang, M.J.; Luo, Y.Y. Adaptive dynamic sliding mode control for overhead cranes. In Proceedings of the IEEE 34th Chinese Control Conference, Hangzhou, China, 28-30 July 2015; pp. 3287-3292. [CrossRef]

24. Wang, W.; Yi, J.; Zhao, D.; Liu, D. Design of a stable sliding-mode controller for a class of second-order underactuated systems. IEEE Proc. Control Theory Appl. 2004, 151, 630-690. [CrossRef]

25. Wang, W.; Liu, X.D.; Yi, J.Q. Structure design of two types of sliding-mode controllers for a class of under-actu-ated mechanical systems. IET Control Theory Appl. 2007, 1, 163-172. [CrossRef]

26. Yang, J.H.; Yang, K.S. Adaptive coupling control for overhead crane systems. Mechatronics 2007, 17, 143-152. [CrossRef]

27. Park, M.S.; Chwa, D.; Eom, M. Adaptive sliding-mode antisway control of uncertain overhead cranes with high speed hoisting motion. IEEE Trans. Fuzzy Syst. 2014, 22, 1262-1271. [CrossRef] 
28. Tsai, C.C.; Wu, H.L.; Chuang, K.H. Intelligent sliding mode motion control using fuzzy wavelet networks for automatic 3D overhead cranes. In Proceedings of the IEEE SICE Annual Conference, Akita, Japan, 20-23 August 2012; pp. 1256-1261.

29. Le, H.X.; Le, A.V.; Nguyen, L. Adaptive fuzzy observer based hierarchical sliding mode control for uncertain 2D overhead cranes. Cyber-Phys. Syst. 2019, 5, 191-208. [CrossRef]

30. Le, V.; Le, H.; Nguyen, L.; Phan, M. An Efficient Adaptive Hierarchical Sliding Mode Control Strategy Using Neural Networks for 3D Overhead Cranes. Int. J. Autom. Comput. 2019, 16, 614-627. [CrossRef]

31. Tuan, L.A.; Lee, S.-G.; Nho, L.C.; Kim, D.H. Model reference adaptive sliding mode control for three dimensional overhead cranes. Int. J. Precis. Eng. Manuf. 2013, 14, 1329-1338. [CrossRef]

(C) 2020 by the authors. Licensee MDPI, Basel, Switzerland. This article is an open access article distributed under the terms and conditions of the Creative Commons Attribution (CC BY) license (http://creativecommons.org/licenses/by/4.0/). 